\title{
Ultrashort laser pulse driven inverse free electron laser accelerator experiment
}

\author{
J. T. Moody ${ }^{*}$ \\ Department of Physics and Astronomy, UCLA, Los Angeles, California 90095, USA \\ and Max Planck Institute for Physics, Munich, Bayern 80805, Germany \\ S. G. Anderson, G. Anderson, S. Betts, and S. Fisher \\ Lawrence Livermore National Laboratory, Livermore, California 94550, USA \\ A. Tremaine \\ Lawrence Livermore National Laboratory, Livermore, California 94550, USA \\ and SLAC National Accelerator Laboratory, Menlo Park, California 94025, USA \\ P. Musumeci \\ Department of Physics and Astronomy, UCLA, Los Angeles, California 90095, USA
}

(Received 17 July 2015; published 29 February 2016)

\begin{abstract}
In this paper we discuss the ultrashort pulse high gradient inverse free electron laser accelerator experiment carried out at the Lawrence Livermore National Laboratory which demonstrated gradients exceeding $200 \mathrm{MV} / \mathrm{m}$ using a $4 \mathrm{TW} 100 \mathrm{fs}$ long $800 \mathrm{~nm}$ Ti:Sa laser pulse. Due to the short laser and electron pulse lengths, synchronization was determined to be one of the main challenges in this experiment. This made necessary the implementation of a single-shot, nondestructive, electro-optic sampling based diagnostics to enable time-stamping of each laser accelerator shot with $<100 \mathrm{fs}$ accuracy. The results of this experiment are expected to pave the way towards the development of future GeV-class IFEL accelerators.
\end{abstract}

DOI: 10.1103/PhysRevAccelBeams.19.021305

The field of advanced accelerators focuses on finding solutions for high gradient acceleration techniques that could replace traditional radio frequency-based accelerating structures which are limited by breakdown to $\sim 100 \mathrm{MeV} / \mathrm{m}$ fields [1]. In laser-based accelerators, short wavelength sources such as high power lasers are employed to exploit high achievable fields while avoiding breakdown. In practice, for a given available energy in the pulse, extremely high laser intensities (and accelerating gradients) can be obtained using ultrashort laser pulse lengths, thereby posing stringent requirements on timing and synchronization with the accelerating electron beams.

Among many different laser-based accelerator schemes, the inverse free electron laser (IFEL) interaction is attractive as the energy exchange between photons and electrons is direct, i.e., occurs in vacuum and far from any boundary (far-field), eliminating the risk of material breakdown altogether. The IFEL scheme accelerates relativistic electrons by drawing power from a laser pulse as the electron

\footnotetext{
*moody@mpp.mpg.de

Published by the American Physical Society under the terms of the Creative Commons Attribution 3.0 License. Further distribution of this work must maintain attribution to the author(s) and the published article's title, journal citation, and DOI.
}

beam and laser pulse copropagate in a periodic magnetic field provided by an undulator magnet.

First investigated by Palmer [2], the IFEL was later proposed for use in high energy physics by Courant, Pellegrini, and Zakowicz [3]. Nearly a decade ago, the STELLA experiment at BNL staged a microbuncher and gap tapered linear undulator to demonstrate high efficiency monoenergetic acceleration with energy gain of up to $9 \mathrm{MeV}$ [4]. The Neptune IFEL experiment at UCLA achieved $20 \mathrm{MeV}$ energy gain and $70 \mathrm{MeV} / \mathrm{m}$ peak gradient with a period and magnetic field tapered undulator and a strongly focused high power $\mathrm{CO}_{2}$ laser [5]. More recently Duris et al. [6] demonstrated $100 \mathrm{MV} / \mathrm{m}$ gradients and high quality beams from an IFEL using helical geometry. Even though due to synchrotron radiation losses, it is widely accepted that the energy reach of the IFEL is limited to $<5 \mathrm{GeV}$, the renovated interest in this laseracceleration scheme comes from the many light source applications in the $1-2 \mathrm{GeV}$ range where scaling is very favorable.

One common element for all these high gradient high gain IFEL accelerators is that they were driven by high power $\mathrm{CO}_{2}$ laser pulses at $10 \mu \mathrm{m}$ wavelength. This is not a mere coincidence as relatively long laser wavelengths are associated with larger transverse spots (mm-size) and longer pulse durations (a few to hundreds of ps) easing the 
alignment and synchronization tolerances in these proof-ofprinciple experiments.

In this paper we discuss a near infrared ultrafast laser pulse driven high gradient IFEL experiment carried out at LLNL where using a strongly tapered planar undulator a $75 \mathrm{MeV}$ beam from an S-band electron linac was accelerated to $125 \mathrm{MeV}$ by a $100 \mathrm{fs} 500 \mathrm{~mJ} \mathrm{Ti}: \mathrm{Sa}$ laser pulse. There are two unique elements in this experiment when compared to previous high gradient IFEL. First, this experiment used the shortest pulse length and the highest laser peak power among all other IFEL experiments to date. As a consequence, we were able to establish new records for energy gain and gradient in IFEL acceleration taking advantage of the order of magnitude larger peak power employed in the experiment. Second, this was the first high gradient IFEL driven by a $800 \mathrm{~nm}$ wavelength commercial Ti:Sapphire based laser system. Due to the large bandwidth of the gain medium, this particular kind of laser technology allows for very short pulse lengths and high peak powers and is now the mainstream for scientific lasers, much more common and compact than high power $\mathrm{CO}_{2}$ lasers employed in previous IFEL experiments.

There is also a more fundamental reason to prefer shorter wavelength laser for high energy IFEL acceleration. In an IFEL maximum energy exchange occurs when the radiation slips over the electron beam by exactly an integer number of cycles every undulator period and the resonant condition is satisfied. For a planar undulator, tuned at the fundamental resonant frequency, this relation can be written as

$$
\gamma^{2}=\frac{\lambda_{w}\left(1+\frac{K^{2}}{2}\right)}{2 \lambda}
$$

where $\lambda$ and $\lambda_{w}$ are the laser and undulator period and $K=0.93 B(T) \lambda_{w}(\mathrm{~cm})$ is the undulator normalized vector potential. Given a maximum magnetic field on the order of $1.5 \mathrm{~T}$, typical of permanent magnet undulator technology, it is easy to see that scaling IFEL accelerators to $\mathrm{GeV}$ energies will require the use of $<1 \mu \mathrm{m}$ laser wavelengths in order to limit the period length to less than $10 \mathrm{~cm}$ (see Fig. 1). While adopting a different undulator technology might alter some of these considerations [7], one should note that shorter undulator periods result in small trajectory offsets $\left(\sim K \lambda_{w} / 2 \pi \gamma\right)$. Since the laser spot size must be at least a few times larger than the particle trajectory wiggling amplitude to ensure good coupling, it follows that shorter period undulators allow tighter focusing and higher laser intensity, and thus maximize IFEL gradient making $<1 \mu \mathrm{m}$ laser wavelengths the preferred choice for GeV IFEL applications.

The paper is organized as follows. First we describe the experimental setup and the original design of the experiment followed by the acceleration results. In the laser section we discuss the implementation of a single shot nondestructive timing diagnostics which was necessary during the optimization phase of the experiment. Due to a

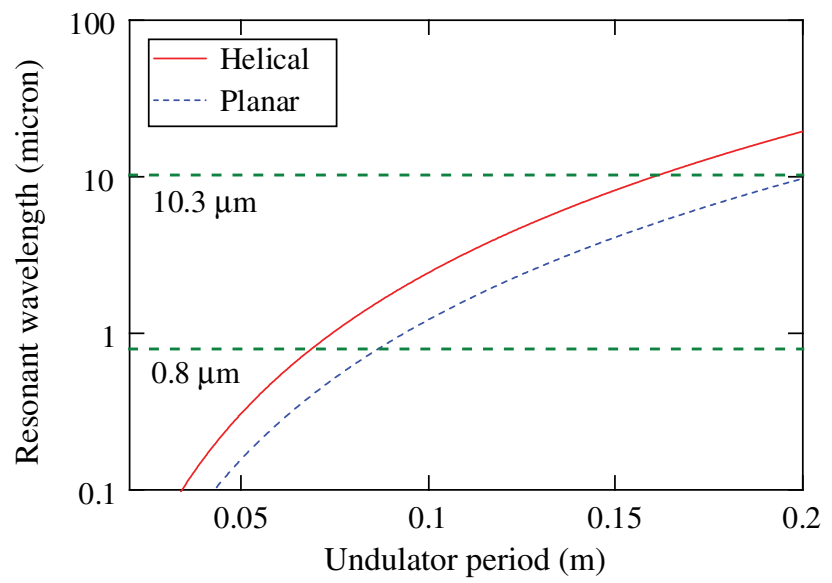

FIG. 1. Resonant energy scaling for fixed magnetic field amplitude.

lower than expected transverse mode quality the energy spectra of the IFEL accelerator were poor and characterized by a rather large energy spread. This is discussed in detail and the measured energy spectra are found in agreement with particle tracking simulations. In the conclusion we discuss the impact of the outcome of the experiment on future IFEL designs.

\section{THE LLNL IFEL ACCELERATOR}

The experiment took place at the LLNL BLDG 194 beamline [8]. The electron source used is a standard SLAC/ UCLA/BNL 1.6 cell S-band photogun. The beam was generated illuminating the cathode with a small portion of the same ultrashort laser system used to drive the IFEL and thus operating the photoinjector in the blowout regime [9]. The beam was then accelerated to $>70 \mathrm{MeV}$ by three $3 \mathrm{~m}$ long SLAC style accelerating sections and finally compressed through a magnetic chicane before focusing into the undulator.

A layout of the interaction area can be seen in Fig. 2 where the magnetic chicane is also shown. The chicane serves also the purpose to couple on the beamline axis the high power laser pulse. The final focus quadrupole magnets bring down the spot size of the electron beam at the center of the undulator to $100 \mu \mathrm{m}$ rms. Spatial overlap between the electron and laser was achieved by monitoring their transverse profiles using standard DRZ $\mathrm{Gd}_{2} \mathrm{O}_{2} \mathrm{~S}$ : Tb fluorescent screens [10] located before, at the center, and after the undulator. A rough temporal overlap within $2 \mathrm{ps}$ is obtained by observing optical transition radiation from the electrons and the laser light on a streak camera. A wide acceptance spectrometer was used after the undulator to measure the beam energy after the interaction. The experimental parameters are summarized in Table I.

The $50 \mathrm{~cm}$ long UCLA/Kurchatov undulator magnet is the same one that was previously used in the Neptune IFEL experiment [11]. In this strongly tapered planar undulator 


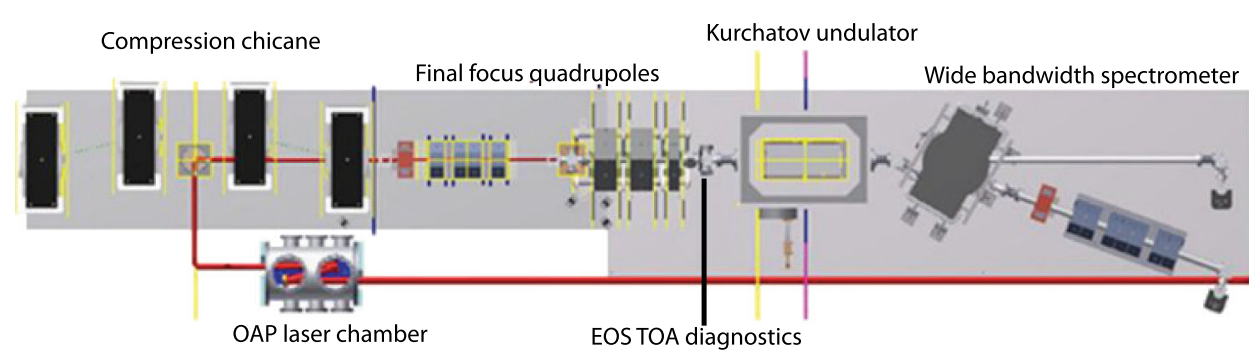

FIG. 2. Diagram of interaction region for the UCLA-LLNL IFEL experiment. The high power Ti:Sa laser (in red) is focused in the OAP chamber, goes through a periscope that preserves its horizontal polarization, and is sent down the beam line using an on axis mirror located the center of the chicane compressor. The electron beam from the S-band linac approaches from the left, is compressed in the chicane, and is focused to a waist at the center of the undulator by two quadrupole triplets.

both the period and magnetic field are increased along the axis to maintain the IFEL resonant condition with a violently accelerating beam. The reduction in the driving laser wavelength (from $10 \mu \mathrm{m}$ for the Neptune experiment to $800 \mathrm{~nm}$ ) results in a $\sqrt{10 / 0.8}$ factor higher resonant energy. The design resonant energy along the interaction assuming a $800 \mathrm{~nm}$ central wavelength driving laser is shown in Fig. 3.

In order for the beam to follow this resonant curve a relatively large ( $>5 \mathrm{TW}$ ) laser power is required. With the aim of minimizing the experimental costs, the laser system for this experiment was specified right at the limit of this threshold. Unforeseen inefficiencies due to power losses in the transport and higher order transverse mode content in the laser transverse profile pushed back the available power by more than a factor of two, effectively preventing full capture and acceleration even for the smallest fraction of the beam.

For a given laser intensity profile, we can calculate the ponderomotive gradient [12] and the undulator tapering

TABLE I. LLNL IFEL design parameters.

\begin{tabular}{lc}
\hline \hline Electron beam & \\
Injection energy & $50 \mathrm{MeV}$ \\
Charge & $50 \mathrm{pC}$ \\
Normalized rms emittance & $2 \mathrm{~mm}-\mathrm{mrad}$ \\
Pulse length after compression (rms) & $100 \mathrm{fs}$ \\
$\sigma_{\text {rms }}$ at focus & $100 \mu \mathrm{m}$ \\
Laser & \\
Wavelength & $800 \mathrm{~nm}$ \\
Pulse length (FWHM) & $100 \mathrm{fs}$ \\
Pulse energy & $500 \mathrm{~mJ}$ \\
Rayleigh range & $7 \mathrm{~cm}$ \\
Focal spot size & $120 \mu \mathrm{m}$ \\
Repetition rate & $10 \mathrm{~Hz}$ \\
Undulator & \\
Undulator period & $1.5-5 \mathrm{~cm}$ \\
Magnetic field amplitude & $0.116-0.686 \mathrm{~T}$ \\
Normalized undulator K & $0.2-2.8$ \\
Gap & $12 \mathrm{~mm}$ \\
Total undulator length & $50 \mathrm{~cm}$ \\
\hline \hline
\end{tabular}

gradient [i.e., derivative of Eq. (1)] along the undulator. In order to have a nonzero area for the trapped region of phase space, the first has to be always larger than the second one. As shown in the Fig. 4, for the actual experimental parameters this is not the case and IFEL resonant acceleration can only be obtained over a distance shorter than the full undulator. Particle tracking simulations confirm that for laser intensities lower than design, particles injected at $50 \mathrm{MeV}$ detrap early in the undulator resulting in modest energy gain and large energy spread.

\section{IFEL ACCELERATION RESULTS}

In order to demonstrate high energy IFEL acceleration of particles, we chose to increase the input beam energy and inject particles further along the resonance curve. This allowed us to verify IFEL acceleration and still measure significant gradient and energy gain even with the smallerthan-design laser intensity delivered to the experiment.

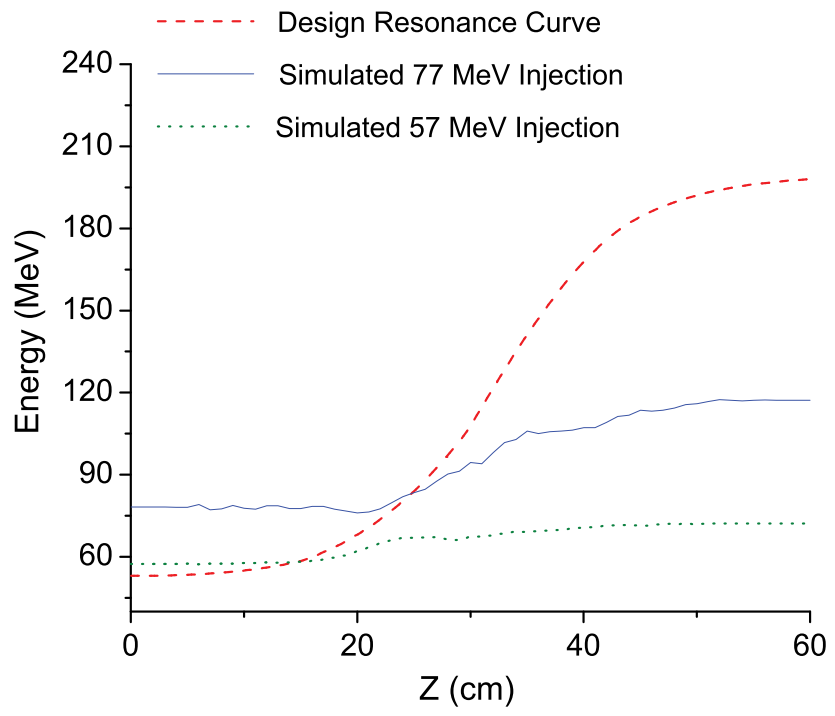

FIG. 3. Evolution along the undulator of the design resonant energy (red-dashed) and of the simulated maximum energy for $57 \mathrm{MeV}$ (green-dotted) and $77 \mathrm{MeV}$ (blue-solid) injection as used in the experiment. 


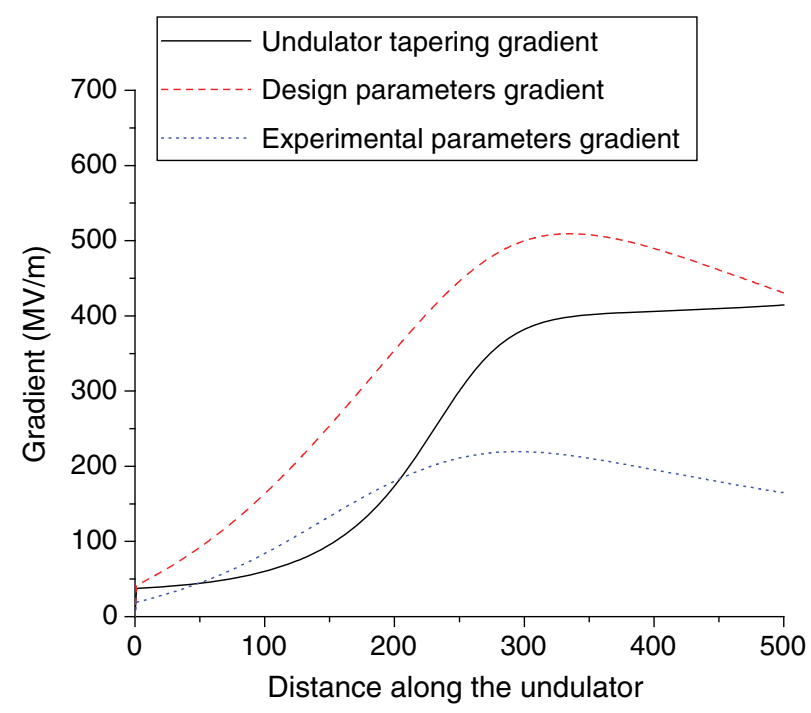

FIG. 4. Ponderomotive gradient using design parameters from Table I and gradient from the undulator resonance condition. The experimental case corresponds to a laser pulse with $50 \%$ lower intensity due to losses in the optical transport and with significant higher order modal content (M2 factor $=1.6$ ).

The evolution of the electron beam in the entire system has been simulated starting from the cathode, through the linac and along the IFEL accelerator using a combination of Parmela [13] for the rf gun and linac, Elegant [14] for the chicane and general particle tracer [15] for the final IFEL interaction. In Fig. 3 we show the evolution of the maximum particle energy for two different input energies (57 and $77 \mathrm{MeV}$ ) as employed in the experiment.

Because the injection energy is higher than the resonant value at the undulator entrance, the energy exchange

$57 \mathrm{MeV}$ Injection Energy

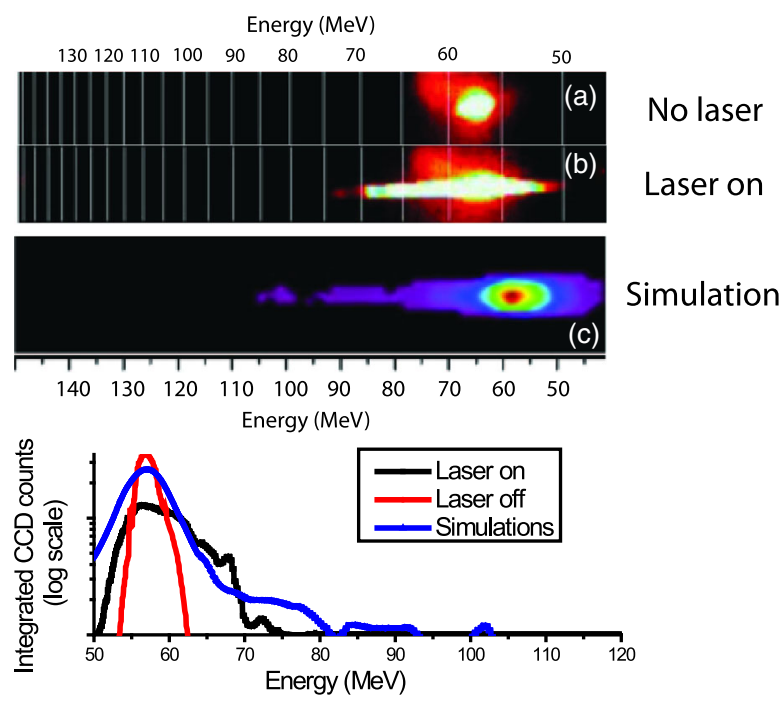

is minimal in the first part of the undulator or more specifically until the longitudinal position where the injection energy intersects the design acceleration curve. For the $77 \mathrm{MeV}$ case we recorded a final energy of $120 \mathrm{MeV}$. According to the simulation $>90 \%$ of the acceleration took place from $20 \mathrm{~cm}$ to $35 \mathrm{~cm}$, resulting in a peak accelerating gradient of $200 \mathrm{MeV}$ per meter. The average gradient over the entire acceleration region can be estimated at approximately $130 \mathrm{MeV}$ per meter.

The measured energy spectra recorded on the spectrometer are shown in Fig. 5 together with the simulated energy spectra. There is fair agreement with the simulation considering the uncertainty in the alignment and in the transverse mode profile of the amplified laser pulse. A $\log$ scale is used to better show the particles at the high end of the energy spectrum. The number of particles accelerated to the maximum energy is smaller than 5\% of the injected electrons, mostly due to the insufficient laser power available and the steep undulator tapering design. Future IFEL experiments should include a prebuncher and a more optimized tapering to maximize the efficiency of energy transfer between laser and electron beam.

\section{SYNCHRONIZATION AND TIMING}

With the electron beam and laser pulse lengths on the same scale as the slippage of the electron beam with respect to the laser along the interaction (i.e., 100 fs time scale), the time of arrival (TOA) jitter between the electron and laser beams can have a significant effect on the performance of the accelerator. To successfully characterize the temporal overlap, the electron beam's TOA with respect to the laser must be

$77 \mathrm{MeV}$ Injection Energy
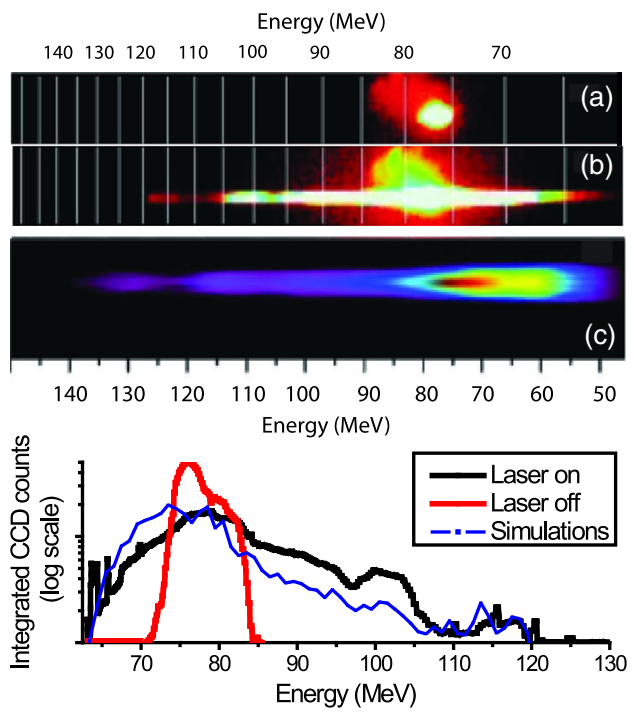

FIG. 5. IFEL spectra for $57 \mathrm{MeV}$ (left) and $77 \mathrm{MeV}$ (right) injection energies. (a) no laser, (b) laser on, (c) GPT simulation results. Integrated lineouts of the spectra are also shown. 


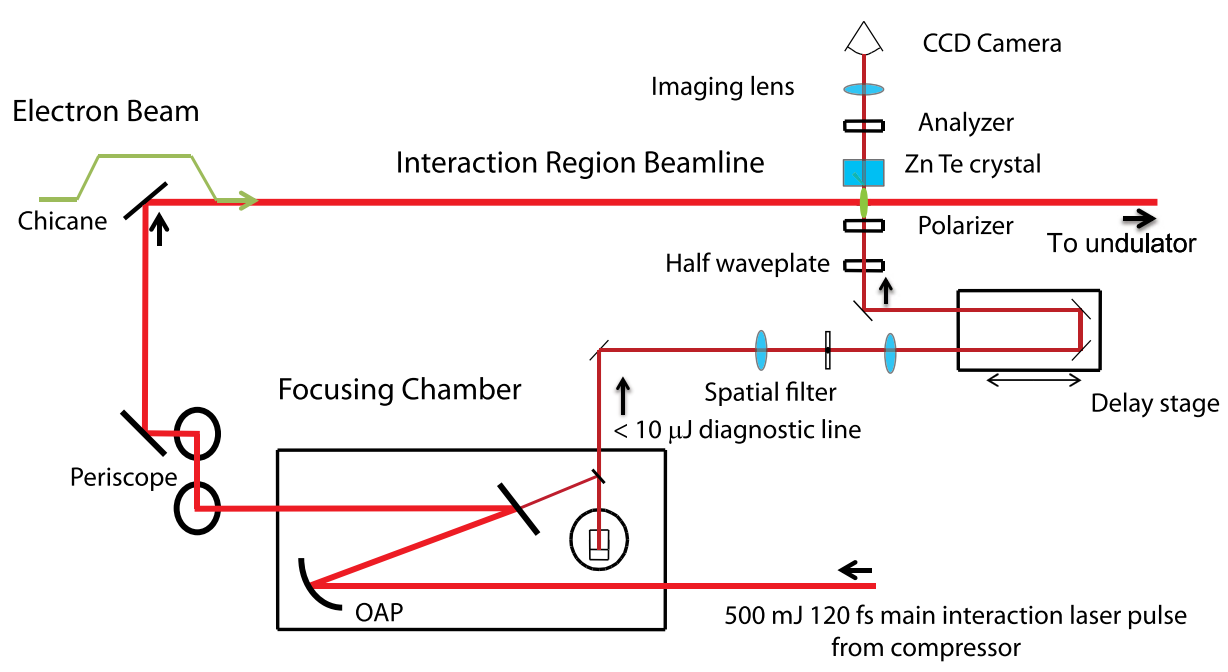

FIG. 6. Diagram of interaction region with details of electro-optic sampling timing diagnostic.

determined for each shot. Therefore we implemented an electro-optic sampling based diagnostic to provide this measurement.

In electro-optic sampling (EOS) a crystal is used to sample the near field of the electron beam which induces a birefringence in the crystal via the Pockel effect. A portion of the main drive laser that powers the optical accelerator is used to probe this index change, resulting in a polarization shift that can be then detected by an analyzing polarizer. There are many ways to read out in a single shot the timing information encoded in the signal [16-19]. In our case, we used the spatially encoded time stamping geometry developed by Scoby et al. [20] for timestamping of pump-probe ultrafast electron diffraction experiments.

The TOA measurement setup is shown in Fig. 6. A small portion of the main drive laser was picked off from the bleed through one of the dielectric mirror, spatially filtered and routed to a $\mathrm{ZnTe}$ crystal located just above the electron beam line axis. A delay stage was used to adjust the temporal overlap of the electron beam and the probe EOS laser at the crystal.

For coincident EOS and IFEL measurements to be captured, the electron beam must be as close to the crystal as possible. Indeed Scoby et al. [20] measured $100 \mathrm{kV} / \mathrm{m}$ with the electron $1 \mathrm{~mm}$ from the surface of the crystal. In the LLNL case, however, the separation between the crystal edge and the electron beam axis must be larger due to the main interaction laser co-propagating with the electron beam to the IFEL undulator. The scattering off the crystal from the transverse distribution tails of the main $500 \mathrm{~mJ}$ pulse is sufficient to saturate the camera. Since the $1 / e^{2}$ transverse spot size of the laser at the crystal plane is $w=3 \mathrm{~mm}$, the crystal must be kept at a distance larger than $\pi w / 2=5 \mathrm{~mm}$ from the axis to prevent saturation of the image and damage of the crystal.

For a relativistic Gaussian electron beam, the radial electric field amplitude at a distance $r$ away from the beam is $E_{r}=\frac{q}{(2 \pi)^{3 / 2} \epsilon_{0} r c \sigma_{t}}$. For a $100 \mathrm{pC}, 200 \mathrm{fs}$ electron beam this gives an estimate for the field at $5 \mathrm{~mm}$ radius from the beam axis of $\sim 1 \mathrm{MV} / \mathrm{m}$ which can induce an easily detectable polarization change.

A representative image from the camera looking at the probe laser after the analyzer is shown in Fig. 7a. The signal shows the characteristic Cherenkov angle of a relativistic electron beam driven wave propagating in the dielectric [20]. The timing information is extracted by determining the position of the peak in a thin slice of the image as shown in $7 \mathrm{c}$. The calibration from pixel to time can be obtained by scanning the delay stage of the diagnostic laser. The injection energy was $57 \mathrm{MeV}$ in this case. The amount of electrons accelerated to energies larger than $65 \mathrm{MeV}$ is strongly dependent on the temporal overlap between the laser and electron beam in the undulator.

Keeping the delay line fixed, we can then acquire multiple images and characterize the time of arrival jitter of the electron beam with respect to the interaction laser prior to running the IFEL experiment. The time of arrival jitter of the electron beam was measured to be $>1 \mathrm{ps,}$ mainly caused by the fluctuations in the amplitude and phase of the high power if system.

When the diagnostic is online at the same time of the laser acceleration experiment, we can use the EOS signal to obtain a time-stamp for each of the IFEL energy modulated electron beam spectra. Two representative shots are shown in Fig. 7. Only when the laser and the electron beam are temporally overlapped inside the undulator a large fraction of electrons are accelerated by the IFEL accelerator. The spectrometer images have been analyzed to extract the maximum energy gain observed by the electrons. Over two hundred correlated images were examined and binned into $200 \mathrm{fs}$ bins. The results of the time of arrival study are reported in Fig. 8 showing the effectiveness of the EOS to capture the timing of the laser accelerator. 


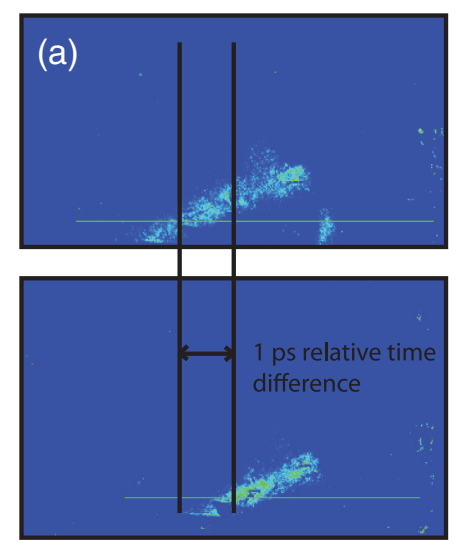

(c)

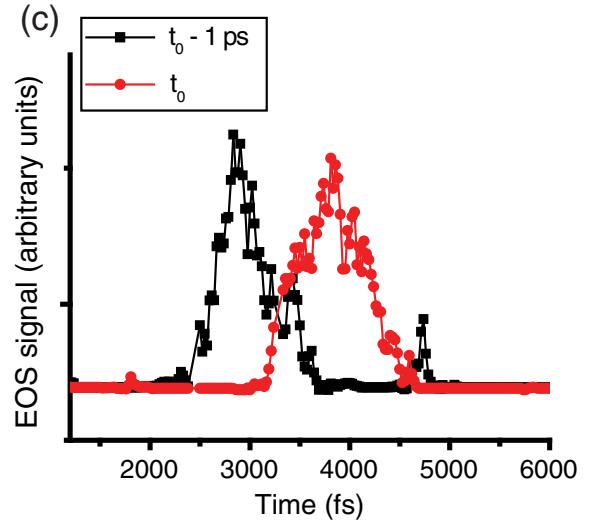

(b)
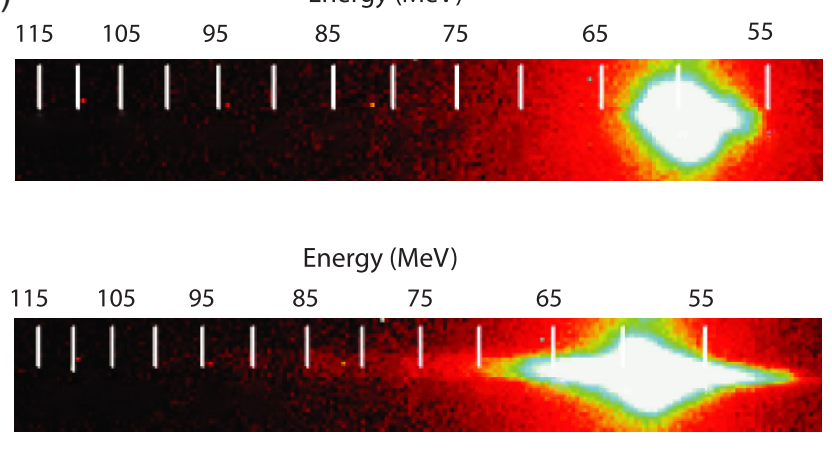

(d) IFEL energy spectrum (65 MeV and above)

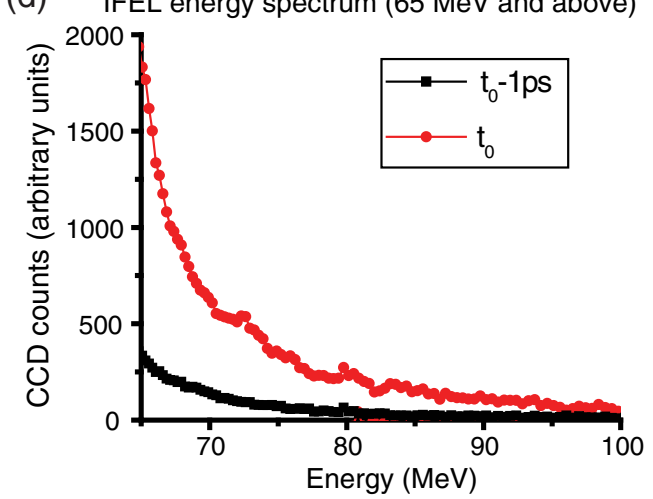

FIG. 7. Coincident EOS images and raw IFEL spectrum images (a and b). Temporal profile of the EOS signal (c) and lineouts of the spectrum images for $>65 \mathrm{MeV}$ electron energy.

A simple cross-correlation model can be used to understand quantitatively the results. Assuming Gaussian profiles for the laser pulse and electron beam temporal profiles with rms duration of $50 \mathrm{fs}$ and 240 , respectively, the model

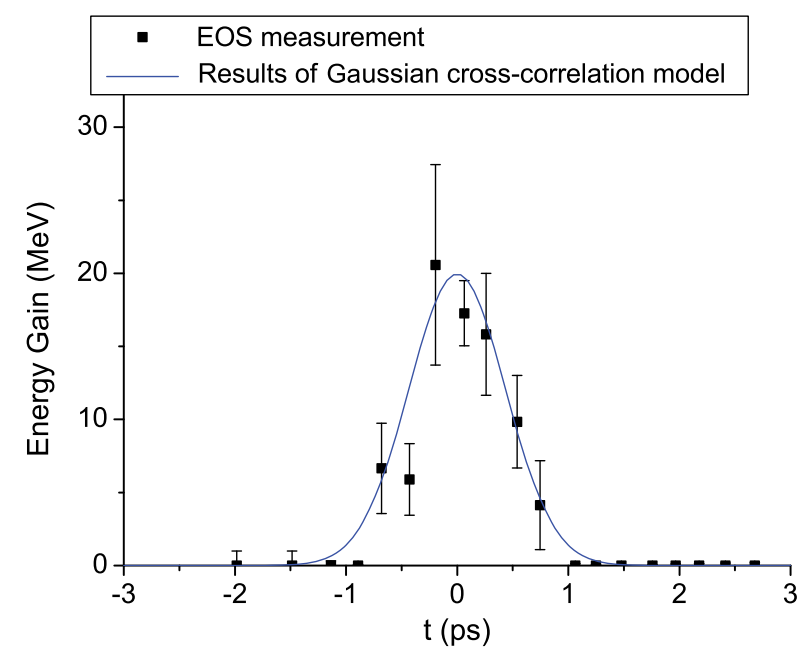

FIG. 8. Results of time of arrival study. Over 200 correlated relative time of arrival and spectrometer images were sampled and binned to provide the temporal overlap profile of the IFEL interaction. The solid curve is the result of the cross-correlation model assuming Gaussian laser and electron temporal profiles with $50 \mathrm{fs}$ and $240 \mathrm{fs}$ rms durations, respectively. predicts a Gaussian timing curve with 250 fs rms duration in good agreement with the measured timing curve for the IFEL interaction.

\section{CONCLUSIONS}

In conclusion, we observed significant IFEL acceleration using a commercial ultrashort pulse $800 \mathrm{~nm}$ laser system resulting in $40 \mathrm{MeV}$ energy gain over approximately $25 \mathrm{~cm}$, with a peak accelerator gradient of $200 \mathrm{MeV} / \mathrm{m}$, paving the way to the use of IFEL accelerators for compact $\mathrm{GeV}$ accelerators for advanced light sources [12].

Due to high peak power and short laser wavelength the demonstration of high energy gain/high gradient accelerator driven by a Ti:Sapph laser system in the UCLA-LLNL IFEL experiment is a key-step along the way of future compact IFEL accelerators. The experiment was mainly aimed at demonstrating high gradient in an IFEL accelerator using short wavelength laser driver. The lack of a prebuncher, the mismatch between design and actual laser parameters prevented full capture and higher efficiency energy transfer.

The LLNL IFEL experiment was not targeting energy transfer efficiency. Among other laser accelerators the IFEL is actually one of the most efficient scheme as no structures and no medium (plasma) can absorb the laser energy. One possibility to improve in efficiency is to accelerate trains of 
electron bunches by recirculating the laser pulse embedding the IFEL in an optical cavity. This possibility originates from the recent results of a recirculated inverse Compton scattering source at the ATF [21] and is subject of active research. In order for a single pass IFEL accelerator to be efficient in transferring the energy from the laser to the electrons as discussed in [12] a much larger $(>1 \mathrm{kA})$ peak current and a prebunched beam must be employed.

Since the laser pulse length was of the same scale as the electron beam slippage with respect to the laser, the temporal overlap was a critical parameter in the experiment and was monitored shot-to-shot using a spatially encoded nondestructive EOS-based diagnostics. As expected, the performance of the IFEL accelerator was observed to be strongly dependent on variation of the relative timing on a scale comparable with laser pulse duration and electron bunch length $(<100 \mathrm{fs})$. Monitoring and controlling the relative timing between high intensity laser pulses and electron beams is a crucial step in improving the reliability of high gradient laser-based accelerator schemes. The timing method employed in this experiment can be easily ported to other laser-based advanced accelerator setups.

\section{ACKNOWLEDGMENTS}

This work was supported by DOE Grant No. DESC0009914:0003. The authors acknowledge J. Duris for helpful comments and suggestions.

[1] H. Braun, S. Dbert, I. Wilson, and W. Wuensch, Phys. Rev. Lett. 90, 224801 (2003).

[2] R. Palmer, J. Appl. Phys. 43, 3014 (1972).

[3] E. D. Courant, C. Pellegrini, and W. Zakowicz, Phys. Rev. A 32, 2813 (1985).

[4] W. Kimura et al., Phys. Rev. Lett. 92, 054801 (2004).

[5] P. Musumeci et al., Phys. Rev. Lett. 94, 154801 (2005).
[6] J. Duris et al., Nat. Commun. 5, 4928 (2014).

[7] J. Harrison, A. Joshi, J. Lake, R. Candler, and P. Musumeci, Phys. Rev. ST Accel. Beams 15, 070703 (2012).

[8] S. Anderson et al., in Proceedings of the 24th Particle Accelerator Conference, PAC-2011, New York, 2011 (IEEE, New York, 2011).

[9] P. Musumeci, J. T. Moody, R. J. England, J. B. Rosenzweig, and T. Tran, Phys. Rev. Lett. 100, 244801 (2008).

[10] R. K. Li, P. Musumeci, H. A. Bender, N. S. Wilcox, and M. Wu, J. Appl. Phys. 110, 174502 (2011).

[11] A. A. Varfolomeev, S. V. Tolmachev, T. V. Yarovoi, P. Musumeci, C. Pellegrini, and J. Rosenzweig, Nucl. Instrum. Methods Phys. Res., Sect. A 483, 377 (2002).

[12] J. P. Duris, P. Musumeci, and R. K. Li, Phys. Rev. ST Accel. Beams 15, 061301 (2012).

[13] L. Young and J. Billen, in Proceedings of the 20th Particle Accelerator Conference, PAC-2003, Portland, OR, 2003 (IEEE, New York, 2003).

[14] M. Borland, Advanced Photon Source LS-287, 2000.

[15] http://www.pulsar.nl/gpt.

[16] G. Berden, W. A. Gillespie, S. P. Jamison, E. A. Knabbe, A. M. MacLeod, A. F. G. van der Meer, P. J. Phillips, H. Schlarb, B. Schmidt, P. Schmser, and B. Steffen, Phys. Rev. Lett. 99, 164801 (2007).

[17] J. Van Tilborg, C. B. Schroeder, C. V. Filip, Cs. Tth, C. G. R. Geddes, G. Fubiani, R. Huber, R. A. Kaindl, E. Esarey, and W. P. Leemans, Phys. Rev. Lett. 96, 014801 (2006).

[18] G. Berden, S. P. Jamison, A. M. MacLeod, W. A. Gillespie, B. Redlich, and A. F. G. van der Meer, Phys. Rev. Lett. 93, 114802 (2004).

[19] A. L. Cavalieri et al., Phys. Rev. Lett. 94, 114801 (2005).

[20] C. M. Scoby, P. Musumeci, J. T. Moody, and M. S. Gutierrez, Phys. Rev. ST Accel. Beams 13, 022801 (2010).

[21] I. Pogorelsky, R. Agustsson, T. Campese, A. Murokh, A. Ovodenko, M. Polyanskiy, and T. Shaftan, J. Phys. B 47, 234014 (2014). 